\title{
EL SENTIDO DEL HUMOR: MÁS ALLÁ DE LA RISA
}

\author{
THE SENSE OF HUMOR: BEYOND LAUGHTER
}

\author{
Jenny Zarela Quezada Zevallos ${ }^{1}$ \\ Lorena Gianino Gadea ${ }^{2}$
}

\section{RESUMEN}

Las autoras realizan una breve revisión sobre la evolución del concepto de humor, los procesos fisiológicos, cognitivos y emocionales, las teorías explicativas sobre el humor, el desarrollo del sentido del humor y la risa, así como la evaluación del sentido del humor y el humor en la psicoterapia, finalizan con investigaciones recientes sobre el sentido del humor.

\section{Palabras clave}

Sentido del humor, risa, desarrollo del sentido del humor

\section{ABSTRACT}

The authors make a brief review of the evolution of the humor concept, the emotional, cognitive and physiological processes, the explanatory theories about the humor, the development of the sense of humor and laughter, as well as the evaluation of the sense of humor and laughter in Psychotherapy, concluding with recent researches about the sense of humor.

\section{Keywords}

Sense of humor, laughter, Psychotherapy

\section{INTRODUCCIÓN}

Nuestro interés en el sentido del humor parte de nuestra experiencia personal en la cual el entrenamiento en la técnica del clown implicó una nueva perspectiva de verse a sí mismo y al entorno de manera más flexible, menos dramática y más juguetona. En nuestro quehacer profesional como psicólogas fuimos incluyendo el uso del humor como una herramienta para favorecer la relación terapeuta-paciente y el proceso terapéutico; es así como nos interesamos por realizar una revisión teórica y de investigación sobre el tema del sentido del humor. En dicha revisión hallamos que la bibliografía en castellano era

1 Licenciada en Psicología por la Pontificia Universidad Católica del Perú. Magíster en Psicología por la Universidad Femenina del Sagrado Corazón. Doctoranda en Psicología en la Universidad Femenina del Sagrado Corazón. Coautora de los libros: Habilidades cognitivas y estilos de aprendizaje: estudio comparativo en menores en crisis en situación de institucionalizados y menores en situación social estable. 2001; Estandarización de Pruebas Psicológicas: áreas cognitiva y de personalidad 2003, Estrategias para desarrollar Habilidades Cognitivas Básicas. 2004. Autora del libro Resúmenes de las Tesis de Psicología 1969- 2008. 2009. Docente en la Facultad de Psicología y Humanidades de la Universidad Femenina del Sagrado Corazón.

2 Licenciada en Psicología por la Universidad Femenina del Sagrado Corazón. Experiencia laboral con niños y adolescentes institucionalizados y niños en edad pre-escolar. Además es clown desde hace 06 años y profesora en la Asociación Bolaroja. 
muy escasa, por lo que tuvimos que recurrir a textos en inglés.

En la investigación realizada descubrimos que el sentido del humor es un constructo complejo y multidimensional que va mucho más allá de una risa. Existen programas para desarrollarlo, técnicas de evaluación, interesantes investigaciones sobre su uso terapéutico. Se piensa que este tema ha sido poco trabajado y ha recibido poca atención por parte de los psicólogos y se confía en que el presente trabajo estimule el interés por este tema.

\section{Evolución del concepto del humor}

En una primera aproximación al término humor, hallamos en el Diccionario de la Lengua Española (2014) siete significados:

Humor. (Del lat. humor, -ōris). 1. m. Genio, índole, condición, especialmente cuando se manifiesta exteriormente. // 2. m. Jovialidad, agudeza. Hombre de humor. // 3. m. Disposición en que alguien se halla para hacer algo. // 4. m. Buena disposición para hacer algo. iQué humor tiene! // 5. m. humorismo (\| modo de presentar la realidad). // 6. m. Antiguamente, cada uno de los líquidos de un organismo vivo. // 7. m. Psicol. Estado afectivo que se mantiene por algún tiempo. buen $\sim 3$.

Las acepciones hacen inicialmente referencia a una manera de ser de la persona, caracterizándola por jovialidad, agudeza, disposición para hacer, decir o mostrar algo de una manera humorística, graciosa, en el sentido del buen humor; como contrario del mal humor que es una actitud con rasgos de agresividad. En ambos casos, esa manera de ser se mantiene en el tiempo.

Coloquialmente se alude a una persona con sentido del humor, como a aquella que muestra frente a las experiencias de la vida, una actitud optimista; muchas veces sacando a relucir el aspecto cómico o gracioso de la situación, también se observa que son capaces de reírse de sí mismas.

Alarcón (2009), en su libro Psicología de la Felicidad, señala que no existe una clara noción de lo que es humor, confundiéndose a menudo con la comicidad y la risa. Reflexiona que el humor puede ser entendido como un "estado" al ser un comportamiento transitorio, cuya duración está asociada a situaciones psicológicas internas, a variables fisiológicas y medio ambientales, relacionadas con el presente; pero también como un "rasgo" afectivo intrínseco de algunas personas, siendo en dicho caso su permanencia más o menos estable.

Este significado del sentido del humor, ha ido cambiando a lo largo de los años. Así Wilhem (1998) hace un análisis del término humor a lo largo de la historia, refiriéndose a estudios realizados por filósofos y psicólogos sobre la estética en los cuales utilizan la palabra cómico, como la facultad de reír o entretener a alguien. Dentro de este término, uno de los elementos es el humor y está definido como la actitud de sonreír frente a la vida y sus imperfecciones y el entendimiento de las incongruencias de la existencia.

Lefcourt (2001) en la revisión histórica sobre el humor halla que diversos filósofos, teólogos $y$ en muchas religiones consideraban la risa o el humor como algo negativo, asociado a la burla o a la maldad frente a los defectos físicos o desgracias percibidos en otros. Al respecto señala que "Discusiones sobre el humor se pueden encontrar en Platón (Philebus), Aristóteles (Poetic), Hobbes (Leviathan) Rousseau (Lettre a M. d'Alembert) estos trabajos se centran sobre todo en la cualidad burlona del reír, afirmando que está mayormente dirigida a las deformidades y fealdades de otros malaintención" (p. 35).

Sin embargo, desde el s. XIII la medicina empieza a descubrir los efectos benéficos de la risa en la salud física de las personas. A partir del s. XIX, en algunas partes de Europa,

No se han considerado las locuciones que se encuentran en el Diccionario de Lengua Española. 
el humor empieza a ser visto como una virtud importante de las personas, así como un estilo de vida. El cambio de un punto de vista negativo a uno positivo del humor y de la risa se hace evidente a partir de los escritos de Sigmund Freud (1905) en Jokes and their relation to the unconscious. Afirma que el humor y la risa permiten la expresión natural del inconsciente. En un artículo posterior sobre el humor en 1928 , lo define como el mecanismo de defensa que permite enfrentar situaciones difíciles sin experimentar una sobrecarga de emociones, distingue tres formas de humor: bromas, lo cómico y el humor.

Una de las respuestas más comunes del humor es la risa, al respecto Martin (2007), en la revisión que realiza sobre la psicología del humor, refiere que ya desde la década del 70 se llegó a la conclusión que la risa es natural al ser humano, después del llanto, es la segunda expresión social que tienen las personas y que esta no es aprendida, observada incluso en niños sordos y ciegos. Asimismo, refiere investigaciones que demuestran evidencias de que tanto el ser humano como los simios presentan la risa como una respuesta natural frente a situaciones de juego o actividades sociales. Por otra parte, se ha demostrado experimentalmente que los chimpancés y gorilas son capaces de desarrollar de manera muy básica el sentido del humor.

El carácter social del sentido de humor ha sido señalado por numerosos autores: Provine (2000) considera que la risa hace ver a una persona como cálida, cooperativa, autoritaria, ineficaz o simplemente fastidiosa. Plantea que los criterios que se utilizan para evaluar si la risa de una persona es apropiada y normal dependerán de la situación y de las características del cómo reímos.

García, B. (2006) sostiene que el humor es un estilo de vida que implica una opción inteligente, un pensamiento racional que, reconociendo la existencia de obstáculos, mantiene una esperanza realista y se dirige hacia los objetivos con la mejor de las disposiciones.

Martin (2011) afirma que el humor es un fenómeno puramente social, porque reímos más fácilmente con otras personas y si reímos solos está relacionado a recuerdos o situaciones que vinculamos a otras personas. Asimismo, señala diversas formas del humor: bromas, humor de las conversaciones espontáneas y humor no intencional. Respecto al humor de las conversaciones espontáneas, Long \& Graesser (1988) citado por Martin (2011) hacen una clasificación más extensa: ironía, sátira, sarcasmo, sobreestimación y subestimación, autodesprecio, burla, respuestas a preguntas retóricas, doble sentido, respuestas inteligentes a declaraciones serias, juegos de palabras y transformaciones de palabras clichés o dichos.

Otras dimensiones del humor que se han estudiado son las diferencias entre la persona que crea las bromas $e$ historias graciosas $y$ las que aprecian el humor, pero son menos capaces de producirlo. También se ha discutido sobre la diferencia entre las funciones del humor, que pueden ser tanto agresivas como defensivas; aunque hay pocos estudios sobre el humor hostil.

Se ha comprobado que existen diferencias entre la preferencia del humor por hombres $y$ mujeres. Al respecto, Hone, Huswitz, Lieberman (2015) confirmaron que los hombres prefieren mujeres que son receptivas a su humor $y$ que las mujeres prefieren hombres que producen humor.

\section{PROCESOS FISIOLÓGICOS, COGNITIVO Y EMOCIONAL}

Frente a una situación de humor se producen un conjunto de procesos de índole fisiológico, cognitivo y emocional los cuales se describirán brevemente a continuación.

\section{Procesos fisiológicos}

El humor está basado en un gran número de procesos biológicos que tienen lugar en el cerebro y en el sistema nervioso. Para experimentarlo, un individuo debe de percibir en un evento elementos de juego $e$ incongruencia. Este proceso perceptual involucra varias regiones del córtex, como las áreas que se encargan de la percepción auditiva y visual, la comprensión del 
lenguaje, cognición social, razonamiento lógico, etc. Una vez que se percibe el humor, este proceso cognitivo estimula sistemas emocionales asociados a emociones positivas involucrando áreas de la corteza prefrontal y el sistema límbico. Estos sistemas emocionales liberan una serie de moléculas bioquímicas que produce cambios en el cerebro y en el cuerpo a través del sistema nervioso autónomo y del sistema endocrino. Además, la activación de emociones positivas, generalmente, es un disparador de expresiones de risa o sonrisa que involucra el cerebro y sus conexiones con los nervios que guían los músculos de la cara y el sistema respiratorio. Martin (2007).

Investigaciones más recientes como las de Swata, Y. y otros (2013) hallaron que en el lado dorsolateral prefrontal del córtex y en el área temporal derecha, el decodificador muestra una clasificación precisa hasta de dos segundos por delante del comienzo del humor. Es decir, los eventos del humor son cifrados en áreas específicas del cerebro cerca de cinco segundos antes de la conciencia de la experiencia de humor.

\section{Procesos cognitivo y emocional}

Koestler (1964) referido por Martin (2007) señala que en el proceso cognitivo se considera la apreciación del humor. Su esencia está en lo incongruente, inesperado y lúdico; características que se hallan en todas las formas de humor. Asimismo "la bisociación ocurre cuando una situación, evento o idea es percibida simultáneamente desde dos coherentes, pero normalmente no relacionadas $e$ incluso incompatibles marcos de referencia "(p. 7).

Morreal (2009) describe el patrón básico del proceso cognitivo frente a una situación de humor:

1. Experimentamos un cambio cognitivo, rápido de nuestras percepciones y pensamientos.

2. Estamos en un modo juego, más que en un modo serio.
3. En vez de reaccionar al cambio cognitivo con sorpresa, confusión, miedo, cólera y otras emociones negativas, lo disfrutamos.

4. Nuestro placer frente a este cambio cognitivo se expresa con la risa, es una señal de que los demás pueden relajarse y jugar.

Con respecto al proceso emocional, Morreal se refiere a las sensaciones que tenemos frente a una situación de humor como una mezcla de sentimientos agradables, de bienestar $y$ de alegría que son una emoción única; sin embargo, no existe una denominación para esa emoción en particular. Ruch, en 1993, propuso una palabra para denominar esta emoción "exhilaratione".

Las funciones psicológicas del humor postuladas por Martin (2007) son:

1. La función cognitiva y social de la emoción positiva de la alegría $y$ felicidad. Se relaciona con una variedad de habilidades cognitivas como flexibilidad cognitiva, mayor creatividad, mejor organización e integración de la memoria, etc. En la parte social, con mayores niveles de responsabilidad social y comportamiento prosocial con expresiones como generosidad y ayuda. Además, las emociones positivas juegan un rol importante en la regulación de relaciones interpersonales fortaleciéndolas y desarrollando la capacidad de agradar a otros.

2. Comunicación e influencia social. El humor siempre tiene un propósito social por ser una forma de comunicar mensajes implícitos que influyen en otras personas. A menudo se lo utiliza para aliviar conflictos entre las personas, para criticar y despreciar a otros.

3. Elaliviodelatensiónyenfrentamiento a la adversidad. Debido a que la 
emoción positiva que acompaña al humor reemplaza a las emociones negativas como ansiedad, depresión o cólera, que de otra manera tendrían lugar frente a una adversidad o situación difícil, permite que la persona piense de manera más flexible y solucione el problema con más creatividad.

\section{TEORÍAS EXPLICATIVAS SOBRE EL HUMOR}

Existen diferentes modelos que explican el sentido del humor como un constructo multidimensional compuesto por diferentes factores. Sin embargo, no existe un solo instrumento que permita evaluar todas sus dimensiones. Kuiper (2004) reseña el modelo de Kirsh y Kuiper (2003) y el de Martin (2003); con respecto al primero de ellos, considera tres dimensiones del humor: la primera es positiva socialmente hábil, se refiere a la capacidad de generar humor de manera espontánea en diversas situaciones sociales, a la facilidad para generar risa en otros y a usar el humor para mantener y reforzar relaciones interpersonales. Las otras dos dimensiones son negativas: una de ellas es el uso agresivo del humor, caracterizado por la falta de preocupación y respeto por otros; es aplicado por personas con estilo mezquino y sarcástico. La tercera dimensión es humor sobrelaborado, el cual consiste en la falta de habilidad para decir o responder ante las bromas o una situación de humor; se caracteriza por un intento inapropiado de encajar a un grupo social utilizando la risa y el humor para agradar a otros y ocultar su propia ansiedad.

El modelo de Martin (2003) menciona cuatro dimensiones, dos adaptativas y dos desadaptativas. Dentro de las adaptativas está el humor de automejora o mejoramiento personal, (adaptativamente autofocalizado), que es la capacidad de mantener una perspectiva humorística incluso en situaciones de estrés o tensión y el uso del humor como una estrategia de afrontamiento. El segundo es el humor afiliativo, (adaptativamente focalizado en otros), que es la capacidad de aumentar o reforzar las relaciones interpersonales, aumentando la identidad y cohesión del grupo, reduciendo los conflictos y reforzando en los otros sentimientos de bienestar. Dentro de las dimensiones desadaptativas, está el humor descalificación personal, (desadaptativamente autofocalizado), es el uso excesivo del humor despectivo hacia sí mismo en un intento inapropiado de ganar la aprobación de otros, este estilo es utilizado para ocultar sentimientos negativos o evitar enfrentarse a un problema. La cuarta es el Humor Agresivo, (desadaptativo focalizado en otros), incluye el ridículo y el sarcasmo. Es utilizado para denigrar a otros, sin tener en cuenta el impacto negativo que puede tener en los demás.

Avner (1984), (referido por Lefcourt), señala las diferencias entre las funciones del humor agresivo y defensivo. El humor agresivo se refiere a menospreciar a otros; mientras que el defensivo alivia el miedo y reduce la respuesta emocional de una experiencia que percibimos como amenazante.

García (2006) plantea un modelo que se compone de cuatro dimensiones:

1. Creación o generación de humor. Se refiere al conjunto de estrategias $y$ habilidades propias para percibir la realidad de forma insólita y comunicarla de modo que provoque risa o sonrisas en los demás.

2. Apreciación del humor y disfrute de la vida. Se refiere a la persona que disfruta riendo, lo hace frecuentemente $y$ se considera alegre. Es el tomarse la vida en serio, pero con toques de humor, pensando en positivo, apreciando el sentido del humor de los demás e incluso riéndose de uno mismo o de sus situaciones.

3. Afrontamiento optimista de problemas. Es el uso del sentido del humor para hacer frente a las dificultades, contratiempos y fracasos de la vida cotidiana. Supone un estilo de pensamiento, un modo de percibir lo positivo que 
tienen todas las personas, cosas o acontecimientos y tratar de actuar en consecuencia.

4. Establecimiento de relaciones positivas. Es la capacidad de utilización del humor como una herramienta para comunicarse $y$ relacionarse socialmente de manera más eficaz.

Este modelo multidimensional parte del postulado de la existencia de una relación directa entre lo que se piensa, lo que se siente y cómo se comportan las personas.

\section{DESARROLLO DEL SENTIDO DEL HUMOR Y LA RISA}

La risa es una manifestación del estado de placer en los bebes, es producida por situaciones de juego como el cosquilleo. Por otro lado, la sonrisa se produce ante una interpretación de los hechos de la realidad.

García (2006) plantea los hitos evolutivos en el desarrollo de la sonrisa y de la risa de la siguiente manera:

Primer mes: los bebés sonríen más durante el sueño REM, también en respuesta al tacto y sonidos suaves al ser mecido, etc. Hay una relación entre sonrisa y liberación de tensión.

Segundo mes (6 a 10 semanas): sonríen ante panoramas de su interés, aparece la sonrisa social (frente a la presencia de la gestalt del rostro humano).

Tercer y cuarto mes: la sonrisa es más frecuente cuando los bebés interactúan con personas, aparece la risa, al inicio como respuesta a estímulos muy activos y posteriormente por discrepancias más sutiles (como los juegos que se realizan con bebés).

Sexto mes: las expresiones de felicidad son cada vez más selectivas, los bebés sonríen más cuando interactúan con personas conocidas y se observan expresiones de tristeza relacionadas con la interrupción grave de la comunicación entre el cuidador y el bebé.

Mc Ghee (1979), (referido por Lefcourt, 2001), realizó un estudio longitudinal en niños durante los 6 primeros años. Parte de la premisa de que el desarrollo del humor se inicia cuando el niño logra reconocer y familiarizarse con los objetos que le rodean, por lo cual las sonrisas y risas anteriores no son indicadores de humor, sino que se originan con la actividad espontánea del sistema nervioso central y con el reconocimiento del rostro humano. Basándose en la teoría del desarrollo cognitivo de Jean Piaget, propone que el desarrollo del humor durante la niñez evolucionaría en cuatro fases:

Primera fase: Acciones incongruentes hacia objetos, surge en el 2 do año de vida en situaciones de juego en las cuales el niño, a sabiendas y por diversión, maneja un objeto como si fuera otra cosa completamente diferente.

Segunda fase: Etiquetas incongruentes hacia objetos y eventos, el desarrollo del lenguaje alcanzado hacia el final del segundo año permite al niño empezar realizar juegos con palabras, tales como renombrar objetos o deliberadamente usar un "nombre incorrecto". Estas manifestaciones muestran una temprana forma de juego y humor a través del uso de símbolos.

Tercera fase: Incongruencia conceptual, es característica entre los tres y cuatro años de edad. Consiste en jugar, por diversión, con los conceptos y sus rasgos rompiendo las categorías conceptuales. Asimismo, en esta fase los niños, al ser muy visuales, también disfrutan al percibir y crear imágenes incongruentes.

Cuarta fase: Múltiples significados, se desarrolla a partir de los 7 años, el niño es capaz de apreciar los acertijos basados en doble significado, disfruta en crear frases y juegos de palabras las cuales son fonéticamente adecuadas, pero abarcan un doble significado. Hacia los 8 años, pueden hacer bromas más inteligentes, contar historias graciosas y el estilo en que 
cuenta empieza a convertirse en un elemento más importante. Este conocimiento de los diferentes significados permite la expresión de la bisociación, que es la superposición de dos ideas o conceptos para originar una nueva.

Mc Ghee realizó estudios longitudinales correlacionales en niños hasta los 6 años y encontró que había conexión entre la conducta maternal y las infantiles tempranas, la risa, y el humor conductual y verbal "niños y niñas con un mayor sentido de humor tienen una historia de muy buenas relaciones positivas con sus madres" (p. 191).

Lefcourt (2001) realizó una revisión de estudios longitudinales sobre humor, concluyó que el cuidado de los niños en los primeros años de vida por padres graciosos y juguetones produce en sus hijos un buen sentido del humor en la adolescencia y temprana adultez.

Para García (2006) la capacidad de pensar con optimismo y expresarse con humor tiene dos grandes influencias. Por un lado, las adquisiciones cognitivas que corresponden a la etapa operacional concreta hacia los 8 años en la que se puede observar si el niño o niña ha desarrollado de modo preferente un estilo explicativo optimista o pesimista ante sus experiencias negativas y positivas. $\mathrm{El}$ otro factor que influye es la inteligencia social que considera habilidades para comprender situaciones como la función de las pistas ingeniosas, son resultado de la interacción social con los padres, otras personas adultas $e$ iguales. En su opinión, los factores que favorecen la enseñanza del optimismo y el buen humor son el modo como se otorga un refuerzo positivo, el refuerzo negativo $y$ el castigo, los aprendizajes por imitación (en especial de sus padres y cuidadores), el estilo de disciplina utilizado. El más eficaz es el inductivo ya que se razona y se explica el alcance de las conductas, las oportunidades que se ofrezcan de experimentar un verdadero control sobre sus vidas y la aceptación que tiene el niño de los padres. Finalmente, señala que para ser padres o educadores con $\mathrm{SH}$ no basta ser alegres o divertidos, requiere también afrontar las adversidades de modo positivo, mantener el humor a pesar de las contrariedades, desarrollar el ingenio, percibir el lado cómico que pueden tener las cosas, así como practicar la amabilidad, suavizando o resolviendo los conflictos sin perder la calma, con la distancia que proporciona el humor (pp. 67-68).

\section{EVALUACIÓN DEL SENTIDO DEL HUMOR}

Existen muchas maneras de evaluar el humor, las cuales pueden ser test, técnicas de contar chistes, test de chistes y dibujos animados, cuestionarios y escalas de tipo autoreporte, informes de iguales y escalas de humor. Se registran hasta un total de 70 según Ruch (1998).

Los más utilizados son los cuestionarios de tipo autoreporte, entre ellos tenemos los siguientes:

- Coping Humor Scale (CHS) Martin \& Lefcourt (1983): es utilizada para evaluar el grado en el cual la persona utiliza el humor como estrategia de afronte al estrés en situaciones vitales potencialmente estresantes. $\mathrm{La}$ escala es de tipo autoinforme, se le plantean afirmaciones frente a las cuales tiene cuatro categorías de respuesta que van de "fuertemente de acuerdo" a "fuertemente en desacuerdo". Consta de 7 ítems.

- Humor Style Questionnaire (HSQ) Martin (2003): evalúa los estilos de humor y considera cuatro estilos, dos de los ellos son adaptativos (humor afiliativo y humor de mejoramiento personal) y dos desadaptativos (humor agresivo $y$ humor de descalificación personal). Cada ítem tiene 7 categorías de respuesta que van desde "totalmente en desacuerdo" a "totalmente de acuerdo". Consta de 32 ítems.

- The situational Humor Response Questionnaire (SHRQ) Martin \& Lefcourt (1984): es una escala de autoinforme, evalúa la frecuencia 
con la que una persona sonríe, ríe o se divierte en situaciones de la vida. Se plantea situaciones frente a las cuales se dan cinco posibles respuestas a cada una, se solicita que se elija la oración que describa de la mejor manera la forma en la que respondería a dicha situación. Consta de 21 ítems.

- Sense of Humor Scale (SHS) Mc Ghee (1994, 1996): evalúa mediante autoinforme y heteroevaluación ocho áreas relacionadas con la conducta del humor (disfrute del humor, seriedad/humor negativo, alegría/humor positivo, risa, humor verbal, encontrando humor en la vida cotidiana, riéndose de sí mismo y humor bajo estrés). Consta de 40 ítems.

- Multidimensional Sense of Humor Scale (MSHS) Thorson \& Powell (1993): evalúa cuatro factores del humor creación del humor, como estrategia de afrontamiento, apreciación y reconocimiento y su uso en situaciones sociales. Se le pide el grado de acuerdo a las afirmaciones de la prueba valorizadas en cinco categorías. Consta de 24 ítems.

- Cuestionario de Autoevaluación del Sentido del Humor (CASH) de García (2005): evalúa mediante autoinforme cuatro dimensiones del sentido de humor (creación, apreciación, afrontamiento optimista de problemas y relaciones positivas). Además dispone de una apreciación general del $\mathrm{SH}$ evaluado por la persona en una escala de 1 a 10 con cuatro categorías de respuesta que van de "casi nunca" a "siempre". Consta de 32 ítems.

- Cuestionario de Heteroevaluación del Sentido del Humor de García, Monjas, Román, Cuetos, Polo y Muñoz (2005). Esta prueba es similar al Cuestionario de Autoevaluación de Sentido del Humor (CASH), con las modificaciones necesarias en la redacción de los ítems, se aplica a un allegada o elegido y se evalúa las cuatro dimensiones del $\mathrm{SH}$.

\section{EL HUMOR EN LA PSICOTERAPIA}

Hay dos posiciones sobre el humor en la terapia: una que favorece el proceso terapéutico porque permite al paciente visualizar una situación desde una perspectiva nueva y diferente, generando también una actitud positiva. Otra posición considera que el humor en la terapia bloquea la libre asociación, y puede ser utilizado por el paciente como una forma de defenderse y protegerse contra el problema sin solucionarlo directamente.

Killinger en el año 1976 realizó un estudio para evaluar si el uso del humor por los terapeutas era una habilidad facilitadora o, por el contrario, inhibía y bloqueaba el proceso terapéutico. Basándose en lo propuesto por Landis y Ross (1933) y posteriormente Koestler (1974) señaló siete formas de uso del humor que aplica el terapeuta:

- Exageración o simplificación

- Incongruencia

- Imprevisto o sorpresa

- Revelación de la verdad

- Superioridad o ridículo

- Represión o liberación

- Juego de palabras

Se esperaría que todas estas categorías produzcan risa, pero se contempló un tipo de humor más sutil, creativo e inteligente que no presentaba una respuesta de risa. De este modo Koestler (1974, citado por Killinger) creó nuevos criterios donde encajaría este humor sin risa:

- Originalidad: la intervención proporciona elementos esenciales de sorpresa por su novedad, exclusividad e inteligencia.

- Énfasis: usa de diversas técnicas continuas como la exageración, 
simplificación o repetición que generan un efecto de tensión acumulada.

- Economía: las intervenciones se hacen de forma implícita en lugar de explícita para lograr la extrapolación o la transposición del contenido.

Entre los principales resultados se encontró que no existe relación entre el adecuado uso del humor por parte del terapeuta y sus años de experiencia. Algunos autores referían que solo los terapeutas con mayor experiencia podrían emplear el humor como una herramienta facilitadora porque sabrían cómo aplicarla en favor del paciente. Sin embargo, en este estudio se vio que no depende de la experiencia sino que hay otros factores que tienen mayor influencia como el nivel de madurez emocional del terapeuta.

Se señala algunas características que posee una persona con un adecuado nivel de madurez: tiene una amplia perspectiva basada en su experiencia de vida que le permite observar su problemática y la de otros en un amplio contexto. Es abierta y flexible, capaz de considerar diversos puntos de vista. Además, es capaz de reírse de sí misma, de aceptar sus limitaciones y las de otros y de ver su propia debilidad desde una perspectiva de humor. Así, si un terapeuta presenta estas características, será capaz de utilizar el humor en sus terapias de manera natural, siempre centrándose en la situación del cliente y no desviando la atención hacia otros aspectos.

Por otro lado, se observó que obtuvieron un puntaje significativamente más positivo las frases "no humorísticas" al ser evaluadas como facilitadoras de la exploración y entendimiento con el cliente, que las que tenían un contenido de humor. Este resultado está relacionado con el hecho de que cuando no se usa el humor hay más frecuencia de formulación de preguntas, lo que contribuye a una exploración más definida del cliente. Mientras que las frases con contenido humorístico no suelen ser a modo de pregunta y requiere un análisis más profundo que va más allá de lo concreto o evidente.
Aún así refieren que la forma en cómo se manifiesta el mensaje, por ejemplo la cualidad de la voz del terapeuta, es más importante que si la frase contiene o no humor, o si se hace en forma de pregunta o no.

Finalmente, a pesar de los diferentes factores que entran a tallar al momento de utilizar el humor en la terapia, se ha encontrado que su potencial se encuentra en su utilización como herramienta que permite que las personas vean su problemática desde una nueva perspectiva. El humor sirve para ampliar la autoconciencia del cliente, mejorando su habilidad para observarse a sí mismo y a otros de manera más objetiva. Tiene mayor utilidad cuando el paciente se siente "atascado" en una situación y percibe su entorno como estresante y abrumadoramente grave. El elemento de sorpresa del humor junto con su cualidad no premeditada, fomentan un cambio progresivo en la perspectiva del paciente y este cambio servirá para desbloquear o aflojar la rigidez del pensamiento.

En otros estudios citados por Alemany y Cabestrero (Idígoras, 2008), Cassel en 1974 hace una revisión exhaustiva de la bibliografía sobre el uso del humor en psicoterapia. Concluye que el humor se convierte en una herramienta muy útil para establecer un diagnóstico y evaluar los cambios producidos durante la terapia. Otro aspecto importante del humor es que puede llegar a ser una estrategia de afrontamiento muy utilizada por personas que presentan alguna dificultad. Greenwald en 1975, basándose en sus propias experiencias como psicoterapeuta, hace hincapié en su importancia como herramienta para lograr insight terapéutico, ya permite la disociación entre un yo que observa y un yo activo al que le suceden las cosas. Para Olson (1976) el humor en la psicoterapia favorece la relación terapéutica, mostrándole al paciente aceptación y respeto. Posibilita que vea sus problemas desde una perspectiva que no había tenido en cuenta antes, además, la risa puede hacerle sentir que tiene cierto control sobre los problemas. De todas maneras, existe bastante responsabilidad por parte del terapeuta en el uso del humor, debe utilizarlo 
con gran destreza y sensibilidad, y en ningún caso, a costa del cliente.

Otros estudios realizados posteriormente tienen resultados similares con respecto al efecto del humor en la psicoterapia.

Alemany y Cabestrero (2008) presentan una síntesis del uso del humor en diversas orientaciones terapéuticas, la se reseña a continuación.

\section{Terapia psicoanalítica}

Es la primera corriente que empezó a analizar la relación entre el humor y el inconsciente. La primera publicación sobre el tema fue hecha por Freud en el año 1905, quien definió el humor como un mecanismo de defensa que utiliza la condensación, la simbolización, el desplazamiento y la represión. Luego otros autores como Vaillant y Buckman refieren que el humor es un mecanismo de defensa adaptativo y el más sano.

Schimel en 1978 indicó que el humor en el psicoanálisis ha sido visto tradicionalmente como una agresión velada y destructiva; pero propuso una reconceptualización del su uso terapéutico. Años más tarde, en 1984, Sands indica que el humor es una forma de expresión emocional que raramente los terapeutas brindan a los pacientes y que el máximo beneficio que se puede obtener de su uso en psicoterapia proviene del propio sentido del humor del paciente. Aunque su uso puede proporcionar al terapeuta un rol más cercano al paciente, facilitando la evaluación de ciertos aspectos de su personalidad y el atravesar sus defensas. Para Korb (1988) las intervenciones humorísticas en psicoanálisis no pretenden evitar las resistencias sino más bien buscar puentes para salvarlas mediante una vía distinta.

Por otro lado, Strean (1994) indica que hay autores que afirman que el uso del humor en terapia supone romper el encuadre terapéutico, provocar una falta de alianza y un signo inequívoco de contratransferencia de parte del terapeuta. Concluye que puede afectar el proceso terapéutico tanto para bien como para mal.

\section{Terapia cognitivo-conductual}

En un principio quizá el rechazo del uso del humor en la terapia de conducta responda a la dificultad que supone el integrar ese fenómeno tan complejo en el paradigma conductual. Koestler (1964) indica que el propósito principal de incluir el humor y la risa en la terapia conductual es mostrarle al paciente una nueva forma de afrontar situaciones que con anterioridad han sido consideradas como un problema. Es así que, por ejemplo, el uso de elementos humorísticos en las jerarquías de la desensibilización sistemática tiene el objetivo de provocar una liberación de tensión que puede ser conceptual o física, dependiendo de si se produce risa o no.

En la terapia racional-emotiva de Ellis, se plantea una variedad de métodos para interrumpiry alterar las creencias irracionales del paciente que actúan como resistencia a los procedimientos terapéuticos. Además, el terapeuta busca reemplazarlas por nuevas $y$ positivas estrategias de afrontamiento. En todo este proceso para vencer las resistencias del paciente y sustituirla, el humor y las estrategias de intención paradójica funcionan especialmente bien, sobre todo con pacientes altamente resistentes y negativos. Otro aspecto que señala este enfoque racional-emotivo es que gran parte de las distorsiones emocionales del paciente se deben a que toma la vida con demasiada seriedad, se exagera el significado y se dimensión de las cosas. Es así como el humor adquiere un gran poder terapéutico al ser una herramienta que ayuda a combatir esta seriedad excesiva.

\section{Terapia de familia y pareja}

Madanes (1987) indica que el propósito del humor en terapia de familia es incluir elementos inesperados o sorpresivos. Pero para que estas intervenciones humorísticas tengan un efecto positivo, el terapeuta debe ser capaz de soportar el ridículo, aparecer como absurdo, ya que en ciertas ocasiones 
la risa puede ir dirigida hacia él. El humor no debe ser confundido nunca con sarcasmo. Es terapéutico reírse con el paciente y no reírse de él. Incluso, es mejor que el terapeuta se ridiculice a sí mismo y no a una familia o una pareja.

Cade (1986) plantea que el humor no debe resultar una imposición, sino debe encontrarse abierto a su uso. El sarcasmo resulta absolutamente desaconsejable, una intervención humorística debe realizarse desde el cariño para que resulte beneficiosa. Además, estas intervenciones de humor deben ir siempre dirigidas a las ideas, a los comportamientos $\mathrm{o}$ a las respuestas de las personas y nunca a ellas misma.

Benninggield (1990) plantea que en el proceso de crecimiento de la pareja es necesario emplear el humor para reactivar la relación, ya que va en contra del aburrimiento que es uno de los principales factores que paraliza el proceso de su crecimiento.

\section{Terapia de grupos}

En una terapia de grupo, Grotjahn (1972) plantea que el terapeuta debe sentirse libre de reír con sus pacientes porque esta reacción muestra al grupo libertad emocional; además, desde ese momento la risa es considerada un signo de libertad.

Bloch, Borwing y McGrath (1983) indican que el humor en las terapias de grupo puede ayudar al terapeuta a mostrar al grupo aspectos de su propia personalidad y a vencer la resistencia. Sin embargo, el humor tiene un efecto negativo cuando es utilizado como defensa frente a la ansiedad, para agredir a otro miembro del grupo o para evitar, de forma frívola, temas importantes. El terapeuta es el responsable de supervisar e impedir que el humor se aplique de esa manera.

Peterson y Pollio (1982) realizaron una investigación sobre el humor en una terapia de grupo. Analizaron las intervenciones humorísticas en tres categorías: las generales, las dirigidas hacia uno mismo y las orientadas hacia otro componente del grupo. Encontraron que las últimas tienden a reducir la efectividad terapéutica; en cambio, las intervenciones dirigidas a personas o instituciones no presentes propendían a aumentar la efectividad de la terapia y las dirigidas a uno mismo generaban un efecto ambiguo.

\section{Programa para mejorar el sentido del humor}

El Programa para mejorar el sentido del humor ha sido elaborado por Begoña García Larrauri (2006). Promueve la utilización de técnicas y estrategias de intervención cognitivo- conductuales, plantea los siguientes objetivos (p. 101):

1. En el aspecto cognitivo, aprender a distanciarse de las preocupaciones y a percibir diferentes alternativas ante un problema o adversidad. Además, Identificar el estilo explicativo pesimista, si existiera, y aprender a rebatirlo.

2. En el aspecto emocional, proporcionar alegría y placer para sentir que la vida merece la pena disfrutarla.

3. En el aspecto conductual, desarrollar habilidades que, desde la expresión corporal hasta la interacción positiva, promuevan el bienestar personal.

El programa propuesto es de preferencia de tipo grupal, puede desarrollarse en trece sesiones con una duración aproximada de 20 horas que se dividen en cinco módulos:

Módulo I. Comunicación, diversión, desinhibición: conformado por actividades cuyo objetivo es provocar la desinhibición, el desbloqueo, la risa en grupo y en general la diversión y facilitación de la comunicación.

Módulo II. Creación de humor: conformado por un conjunto de actividades que buscan desarrollar el ingenio, la creatividad y la imaginación utilizando técnicas de papel y lápiz, expresión corporal o práctica diaria de una actitud receptiva y permeable al humor. 
Módulo III. Apreciación de humor: las actividades tienen como objetivo recuperar el humor lúdico, favorecer el cuidado emocional, el lenguaje positivo, la relajación, el disfrute del presente, etc.

Módulo IV. Afrontamiento optimista de adversidades: se busca aprender a lidiar con los contratiempos, bien sea a través de la prevención o de la detección de creencias pesimistas o irracionales, modificándolas y modelando un estilo explicativo optimista.

Módulo V. Relaciones positivas con humor: se busca con estas actividades promover el agradecimiento como medio de obtención de satisfacción con el pasado y el presente, estimular un cambio positivo en las relaciones al dar y recibir, etc.

García (2006) informa de los resultados de la aplicación del Programa para mejorar el humor, en personas adultas (entre 45 y 68 años, con una media de 54 años) de ámbito urbano, diseño preexperimental, un solo grupo. Los efectos son aumento del interés general por el $\mathrm{SH}$, incremento de la sonrisa, risa y humor en la vida diaria, promover un incremento del gusto por el juego, contribuir a la pérdida del sentido del ridículo y fomentar una actitud mental positiva, entre los más importantes.

En el caso de la aplicación del programa a personas mayores (entre 47 y 72 años, con una media de 59 años) de ámbito rural, de diseño cuasi-experimental, un solo grupo, se informa mejoras del sentido del humor y en el bienestar personal de los sujetos. Asimismo se aplicó el programa en estudiantes universitarios (edad promedio 21 años), diseño experimental, pretest-postest, con dos grupos, se halló diferencia estadísticamente significativa en el bienestar subjetivo de los sujetos del grupo experimental y una mejoría en las variables: creación del humor, apreciación del humor y bienestar psicológico.

Finalmente, se aplicó en el ámbito escolar, se utilizó el sentido del humor como recurso metodológico para la enseñanza de la lengua inglesa y la educación artística $e$ indirectamente para el desarrollo de actitudes mentales positivas en el alumnado de primaria. La experiencia se realizó con 125 estudiantes con edades comprendidas entre los 6 y 11 años que cursaban desde primero hasta quinto de primaria. Los resultados presentan un incremento en el rendimiento de las asignaturas seleccionadas, de la motivación y del grado de satisfacción en el alumnado, así como repercusiones en otros ámbitos de la vida escolar, de otras variables relacionadas con $\mathrm{SH}$ en los niños (as), creatividad, relaciones intragrupo y con la profesora, conductas más alegres, etc.).

\section{Investigaciones recientes sobre el sentido del humor}

Algunas investigaciones muestran el efecto que tiene el humor en la salud. Cann y Kuiper (2014) en una entrevista sostienen que el sentido del humor es un constructo multidimensional que incluye varios componentes cuyos efectos pueden ser benéficos o perjudiciales para el bienestar. Además, Cann, A. y Collete, Ch. informaron, en un estudio que realizaron ese mismo año, encontraron que los afectos estables están relacionados a la resiliencia y al bienestar psicológico y el sentido del humor de tipo Mejoramiento personal está relacionado con afectos más estables y negativamente a afectos negativos estables. A través de los afectos positivos influye en la resiliencia, bienestar y el distrés. En otro estudio, Bennett y otros (2008) realizaron una revisión sobre resultados de investigaciones de efectos del humor y de la risa en la salud, concluyeron que influyen en la tensión muscular, la función cardio-respiratoria y varias medidas fisiológicas del estrés.

Se han realizado intervenciones terapéuticas basadas en el humor a diversos grupos de personas, por ejemplo, en niños. Es el caso de una investigación sobre los efectos de los clowns en niños hospitalizados por patologías respiratorias. En el post-test se halló efectos favorables en la presión arterial, en la frecuencia respiratoria y en la temperatura, así como en la presión sistólica y frecuencia cardiaca (Bertini 2011). En un grupo de adultos se realizó este tipo de intervenciones enfocado en sus efectos, 
en el alivio de dolores crónicos. En ancianos, después de la aplicación de un programa de humor de ocho semanas de duración, se halló una significativa disminución del dolor y de la percepción de tristeza y un significativo incremento en la felicidad y satisfacción de vida (Tse y otros, 2010). Kopytin y Lebeder (2013) informan de un estudio realizado con veteranos de guerra en el cual se logró incrementar el humor en el grupo experimental por medio de una estrategia basada en el dibujo con énfasis en el uso del humor. Un estudio con pacientes ancianos con depresión realizado por Konradt, B. y col. (2013) informa sobre la eficacia de la terapia del humor estandarizada en los participantes de su grupo, que mostraron cambios en el estado de severidad y en el nivel de satisfacción con la vida. Se han realizado también este tipo de intervenciones en pacientes con esquizofrenia. Cai, Ch. y col. (2014), los autores informan sobre un estudio en el que se entrenó habilidades de humor en pacientes esquizofrénicos de un servicio de salud mental y se halló mejoras en los proceso de rehabilitación y en el sentido del humor.

Otros estudios se han focalizado en determinar las características del sentido del humor en pacientes diagnosticados con Autismo. Samson (2013) halló que las personas con este espectro pueden apreciar los estímulos humorísticos como bromas y chistes si estos no son complejos. En otro trabajo se informa puntajes bajos en el rasgo de alegría y altos en el rasgo de seriedad que son elementos descriptores de la susceptibilidad al humor. Así mismo puntuaron bajo en las escalas relacionadas con la comunicación social (humor afiliativo, humor de entretenimiento) y retrataron un estilo de humor más socialmente frío. También obtuvieron puntajes bajos en la capacidad para percibir el significado humorístico de un evento y en el uso del humor de mejoramiento personal.

En nuestro país se han realizado las siguientes investigaciones:

Zavala (1999) realizó un estudio de tipo preexperimental y expost-facto sobre el sentido del humor adaptativo (SHA) en niños institucionalizados entre 7 y 11 años desde el enfoque de la resiliencia. Halló una alta asociación (gamma $=0.67$ ) entre rendimiento escolar y SHA, entre SHA y autoconcepto (gamma $=0.69$ ), entre SHA y creatividad medida a través de las artes plásticas (gamma= 0.82). Las estrategias de afronte usadas por los niños con alto SHA se centran en aquellas en las que asume una actitud proactiva y busca la relación con otro, la más usada es la de buscar a otra persona. Finalmente, encontró alta correlación entre SHA y la capacidad lúdica en las dimensiones de capacidad de simbolismo, personificación, movimiento, fluidez ideativa, flexibilidad, plasticidad, estructura coherente y persistencia en el juego.

Por su parte, Cassaretto \& Martínez (2009) realizaron la validación de la Escala del Sentido de Humor (HSQ) de Martin y col. (2003) en un grupo de estudiantes universitarios. Hallaron una escala confiable con coeficientes entre 0.64 y 0.78 y una estructura factorial que confirma la existencia de cuatro factores que explican el $42.94 \%$ de la varianza total. Asimismo, encontraron correlaciones entre bienestar psicológico y esperanza con las áreas de afiliación y mejoramiento personal de la HSQ son positivas y significativas, los cual apoya la validez convergente y de constructo de la escala.

Tateishi (2011) investigó, en un grupo de pacientes mujeres con cáncer de mama entre los 37 y 65 años, la relación entre estilos de humor y sintomatología depresiva y halló una asociación negativa entre el estilo de afiliación $(p=0,32)$ y la sintomatología depresiva, y una asociación positiva con el estilos agresivo $(p=$ 0,32 ). También encontró asociaciones entre los estilos de humor y variables como ocupación, estadio clínico, recibir hormonoterapia y tener enfermedades adicionales al cáncer.

\section{CONCLUSIONES}

1. El concepto de humor ha evolucionado históricamente, desde ser considerado como algo negativo asociado a la burla a un fenómeno complejo el cual es un rasgo de la personalidad y es básicamente una actitud positiva frente a la vida y sus problemas, caracterizada por quitar peso a las situaciones cotidianas y considerarlas 
desde una perspectiva más divertida. Sin embargo, también existen formas de humor como la ironía, la sátira, el sarcasmo, etc. cuyos efectos sociales son definidos como negativos.

2. El fenómeno del humor abarca procesos fisiológicos que tienen lugar en el cerebro y en el sistema nervioso. Están asociados con áreas relacionadas de percepción auditiva y visual, de percepción del lenguaje, de cognición social y de razonamiento lógico. También activan emociones positivas que se expresan a través de la risa o sonrisa. Los procesos cognitivos frente a una situación de humor conforman un patrón básico partiendo de un cambio cognitivo, un paso a un modo juego, un reaccionar al cambio cognitivo con disfrute que se expresa con la risa, lo que produce relajación y juego.

3. El humor tiene funciones psicológicas importantes en relación con habilidades cognitivas como flexibilidad cognitiva, creatividad, y funciones sociales en las cuales las emociones positivas, despertadas por el humor, juegan un rol importante en la regulación de relaciones interpersonales al poder agradar a otros. Asimismo, la función del alivio de la tensión y enfrentamiento a la adversidad hace que se considere al humor como una herramienta de enfrentamiento al coping.

4. Con respecto a las teorías explicativas sobre el humor, todas coinciden en que es un constructo multidimensional compuesto por diferentes factores, algunos modelos puntualizan las dimensiones relacionadas con el uso positivo y negativo del humor y la habilidad para responder a las bromas (Kirsh y Kuiper, 2003). Otro modelo es el de Martin (2003), menciona cuatro dimensiones, dos adaptativas y dos desadaptativas. Por último García B. y col. (2006) plantean un modelo con dimensiones en relación a la creación, apreciación del humor, afrontamiento optimista de problemas y establecimiento de relaciones positivas. Este modelo postula la existencia de una relación directa entre lo que se piensa, lo que se siente y cómo se comportan las personas en relación al humor.

5. El desarrollo del sentido del humor se inicia en el niño desde la risa y la sonrisa para posteriormente durante la niñez transcurrir por fases de la percepción del humor, según el modelo de Mc Ghee (1979).

6. Existen muchas maneras de evaluar el humor, las cuales pueden ser test, técnicas de contar chistes, test de chistes y dibujos animados, cuestionarios y escalas de tipo autoreporte, informes de iguales y escalas de humor, registrándose hasta un total de 70 según Ruch, W. (1998). Hemos reseñado las más frecuentemente usadas en la investigación.

7. Con respecto al humor en la psicoterapia existen dos posturas: una que considera que es un facilitador y la otra como un interferente en la libre asociación. El uso del humor por el terapeuta tiene múltiples formas como la exageración, la incongruencia, el imprevisto o sorpresa, el juego de palabras, entre otras.

8. El uso adecuado del humor por parte del terapeuta tiene como uno de sus factores más importantes su nivel de madurez emocional, lo que permitiría aplicarlo en sus terapias de manera natural $y$ centrándose en la situación del paciente.

9. Los enfoques terapéutico-psicoanalítico, cognitivo-conductual, terapia de familia y pareja y terapia de grupos utilizan de modos distintos el humor buscando facilitar en el paciente o grupo mayor fluidez en la expresión de sus emociones y una visión diferente de su situación.

10. Las investigaciones sobre el sentido del humor muestran el efecto que tiene en la salud y su aplicación en las intervenciones terapéuticas en diferentes grupos de personas como niños, ancianos, veteranos de guerra, pacientes con esquizofrenia por mencionar algunos. 


\section{REFERENCIAS}

Alarcón R. (2009) Psicología de la felicidad. Lima: Universidad Ricardo Palma.

Alemany, C. y Cabestrero, R. (2008) Humor, psicología y psicoterapia: estudios e investigaciones, en El valor terapéutico del humor. Idígoras, A. (3 Edición). Bilbao: Desclée de Brower.

Bennett, M. y Lengacher, C. (2008) Humor and laughter may influence health: III laughter and health outcomes. Evidence-based Complementary \& Alternative Medicine. March, N. ${ }^{\circ}$ 5, Vol. 1, 37 - 40.

Bertini, M., Isola, E., Paoli, G. y Curcio, G. (2011) Clowns benefic children hospitalized for respiratory pathologies. Evidence-based Complementary \& Alternative Medicine. June, N. ${ }^{\circ} 8$, Vol. 1, 1 - 9. DOI: 10.1093/ecam/neq064.

Cai, Ch., Yu, L., Ronh, L. y Zhony, H. (2014) Effectiveness of humor interventions for patients with schizophrenia: a randomized controlled trial. Journal of Psychiatric Research. December, N. ${ }^{\circ}$ 59, 174 - 178. DOI: 10.1016/j.psychires2014.09.010.

Camacho, J. (2008) El humor, en Casullo, M. (compiladora) Prácticas en Psicología Positiva Buenos Aires: Lugar Editorial. 111 - 136.

Cann, A. y Collete, Ch. (2014) Sense of humor, stable affect an psychological well-being. Europe`s Journal of Psychology. N. ํ 10, Vol. 3, 464 - 479. DOI: 10.5964/ejop.v10i3.746

Cann, A., Kuiper, N. (2014) Research on the role of humor in well-being and health. Europe`s Journal of Psychology N. ${ }^{\circ}$ 10, Vol. 3, 412 - 428.

Cassaretto, M. y Martínez, P. (2009) Validación de la Escala del sentido de humor en estudiantes universitarios, en La Revista de Psicología N. ${ }^{\circ}$ 27, Vol. 2, 287 - 307.

García - Laurrauri, B. (2006) Programa para mejorar el sentido del humor. iPorque la vida con buen humor merece la pena! Madrid: Pirámide.

Hone, L., Huswitz, W. y Lieberman, D. (2015) Sex differences in preferences for humor; a replication, modification an extension. Evolutionary Psychology N. ${ }^{\circ}$ 13, Vol. 1, 167 - 181.

Killinger, B. (1987) Humor in psychotherapy: a shift to a new perspective. En Handbook of Humor and Psychotherapy: Advances in the clinical use of humor. Fry, W. and Salameh, W. Sarasota: Professional Resource Exchange, Inc.

Konradt, B., Hirsch, R., Jonitz, M. y Junglas, K. (2013) Evaluation of a standardized humor group in a clinical setting: a feasibility study for older patients with depression. International Journal of Geriatric Psychiatry. August, N. ${ }^{\circ}$ 28, Vol. 8, 850 - 857.

Kopytin, A. y Lebeder, A. (2013) Humor, self-attitude, emotions and cognitions in group art therapy with war veterans. Art therapy: Journal of the American Art Therapy Association N. ${ }^{\circ} 30$, Vol. 1, 20 - 29.

Kuiper, N., Grimshaw, M., Leite, C. y Kirsh, G. (2004) Humor is not always the best medicine: specific components of sense of humor and psychological well-being. Humor N. ${ }^{\circ} 17(1 / 2)$, 135-138. 
Lefcourt, H. (2001) Humor. The psychology of living buoyantly. New York: Springer Science+Business Media, LL.

Madanes, Cl. (1987) Humor in strategic family psychotherapy. En Handbook of humor and psychotherapy. Advances in the clinical use of humor. Fry, W. and Salameh, W. Sarasota: Professional Resource Exchange, Inc.

Martin, R. (2007) The psychology of humor. An Integrative Approach. United State of America: Elsevier Academic Press.

Morreal, J. (2009) Comic relief. A comprehensive philosophy of humor. Malasia: Wiley - Blackwel Paredes G. D. (2008) La experiencia del humor en personas con discapacidad intelectual. Revista Electrónica de Investigación Psicoeducativa N. ํ 14, Vol. 6, 201 - 208.

Provine, R. (2000) Laughter a scientific investigation. New York: Penguin Group.

Ruch Willibald (1998) Foreword and overview Sense of humor: a new look at an old concept, en Ruch Willibald (edit) (1998) The sense of humor. Explorations of a personality characteristic. Berlín: Mouton de Gruyter. 3-14

Real Academia Española (2014) Diccionario de la Lengua Española. 23 Edición. Disponible en Internet http://www.rae.es/recursos/diccionarios/drae.

Samson, A. (2013) Humor (lessness) elucidated-sense of humor in individuals with autism spectrum disorders: Review an introduction. Humor International Journal of humor research. July, N. ${ }^{\circ}$ 26, Vol. 3, 393 - 409.

Samson, A., Huber, O. y Ruch, W. (2013) Seven decades after Hans Asperger`s observations: a comprehensive study of humor in individuals with Autism Spectrum disorders. Humor International Journal of humor research. July, N. ${ }^{\circ} 26$, Vol. 3, 441 - 460.

Sawata, Y., Komuno, K., Morita,T. y Hirumo, N. (2013) Decoding humor experience from brain activity of people view comedy movies. PLos ONE. December, N. ${ }^{\circ}$ 8, Vol. 12,1 - 9.

Tateishi, V. (2011) Estilos de humor y sintomatología depresiva en pacientes con cáncer de mama. Tesis de Licenciatura Psicología Clínica. Lima-Perú: Pontificia Universidad Católica del Perú.

Tse, M., Lo, A., Cheng, T., Chun, E., Chan, A. y Chun, H. (2010) Humor therapy: relieving chronic pain and enhancing happiness for older adults. Journal of aging research, 1 - 9.

Zavala, M. (1999) Sentido de humor adaptativo en niños institucionalizados desde el enfoque de la resiliencia. Tesis de Licenciatura en Psicología con mención en Psicología Clínica. Lima-Perú: Pontificia Universidad Católica del Perú.

Fecha de recepción: 26 de marzo 2015

Fecha de aceptación: 28 de abril de 2015 